\title{
Three-month Effects of Immunotherapy on Clinical Features and Pulmonary Function Tests of Patients with Allergic Rhinitis Concomitant with or without Asthma
}

Guzin Ozden', [MD]

ORCID: 0000-0003-4856-2267

Pelin Duru Cetinkaya ${ }^{2}$, [MD]

ORCID: 0000-0002-4428-8590

\section{sel) ABSTRACT Con}

Objective: We aimed to evaluate our patient's treatment responses to three-month immunotherapy for allergic rhinitis with or without asthma, and to investigate the factors affecting treatment

Material and Methods: A total of 53 patients receiving subcutaneous immunotherapy for allergic rhinitis with or without asthma were included in the study. All patients were positive for mite skin prick test. Asthma was present as a concomitant disease in 28 (52.8\%) patients. Patients with and without asthma were classified in two groups as "Allergic rhinitis + Asthma+" and "Allergic rhinitis+ Asthma-". The values of pulmonary function tests of all patients, and in rhinitis with asthma patients, the Asthma Control Test scores before and after three months of treatment were recorded.

Results: In "Allergic rhinitis + Asthma+" and "Allergic rhinitis + Asthma"groups, male/female ratio was $7 / 21$ and $10 / 15$, mean age was $33 \pm 9$ and $32 \pm 9$ years, respectively. No statistically significant differences were present between the pre-treatment and 3-month-treatment values of pulmonary function test. The difference between the pre-treatment and 3-month treatment asthma control test scores was strongly significant.

Conclusion: By using pulmonary function test in allergic rhinitis and asthma control test in asthma, we showed that the effectiveness of allergen specific immunotherapy started in three months, which was earlier when compared to the current literature. When asthma is associated with allergic rhinitis, which is quite common, the control of asthma is also provided with the treatment of allergic rhinitis.

Keywords: Allergic rhinitis, asthma, immunotherapy, respiratory \section{function tests}

Corresponding Author: Guzin Ozden

Adana City Training and Research Hospital, Allergy

Immunology Clinic, Adana, Turkey.

E-mail: drkocamazguzin@hotmail.com

Received: 4 October 2020, Accepted: 11 November 2020,

Published online: 31 December 2020

\section{INRODUCTION}

Allergic rhinitis (AR) is an inflammatory disorder occurring due to exposure of the mucosa to allergens and manifesting with symptoms of nasal itching, discharge, congestion, and sneezing. The prevalence of AR is estimated as $20 \%-40 \%$ throughout the world [1], and studies conducted in our country have revealed various ratios such as $14.5 \%$ and $22.7 \%$ [2, 3]. AR is classified as persistent and intermittent. Persistent AR is defined as the presence of rhinitis symptoms for over four days of a week and consecutive four weeks of duration.

Asthma is a variable airway obstruction occurring because of chronic inflammation and manifesting with symptoms of cough, wheezing, and chest tightness. These symptoms are associated with bronchoconstriction, airway thickening and increased mucus. In patients with rhinitis, the 
incidence of asthma has been reported to change between $10 \%$ and $40 \%$ [4]. Even though asthma may develop in all patients with rhinitis, the probability of development is higher in patients with moderate-to-severe rhinitis compared to those with mild and intermittent rhinitis $[4,5]$. Moreover, the presence of rhinitis in most patients with asthma supports the concept of "one airway, one disease" [6].

Immunotherapy is the only treatment option that can change the progression of the disease [7]. Immunotherapy has been shown to be effective in both allergic rhinitis and asthma [8]. This study aimed to evaluate the first 3-month treatment responses of the patients in whom we started immunotherapy with the diagnosis of allergic rhinitis.

\section{MATERIAL and METHODS}

The approval of the Local Ethics Committee was obtained before initiation of the study (\#2016/48). Fifty-three patients aged between 18 and 54 years, who were receiving mite-specific allergen immunotherapy (ALK 510, Allergo 708/725) for their diagnosis of allergic rhinitis with or without asthma, were included in the study. We diagnosed asthma according to the evidence of variable expiratory airflow limitation and history of variable respiratory symptoms (GINA 2019) [9]. Informed consent of the patients was received before they participated in the study. The criteria to include in the study were being over 18 years of age and being diagnosed with persistent rhinitis. The exclusion criteria were being under 18 years of age, being pregnant, a history of hospitalization in the last month, a change in treatment because of an exacerbation of asthma in the last month, and the forced expiratory volume in one second (FEV1) value below $70 \%$.

All patients started mite-specific immunotherapy in fall (September, October, and November). For three months, they received weekly subcutaneous immunotherapy (SCIT). All patients received one antihistamine drug in a day. Patients with rhinitis and asthma received regular daily ICS (inhale corticosteroid)-formaterol (GINA step 2). None of them had had an exacerbation of asthma before SCIT. Pulmonary function tests (PFT) were performed before initiation of SCIT and recorded. PFTs were performed again following three months of treatment. PFTs of all patients were performed by the same nurse and the same device (Zan 100). The PFT results before SCIT, and the PFT results following three months of SCIT were compared to each other.

Patients were divided into two groups. One group was the association of allergic rhinitis and asthma $(\mathrm{AR}+\mathrm{AST}+)$, and in the other group, allergic rhinitis was not accompanied by asthma (AR+AST-). The demographic characteristics, smoking history, family history of asthma and other disorders, and physical examination findings of the patients, the total Ig E results, and the phadiatop (immunoCAP 100) test results were recorded. The skin prick tests and allergen-specific immunotherapy were performed by the same allergist and recorded. An asthma control test (ACT) was performed for the patients in the group with allergic rhinitis and asthma. ACT is an internationally proven test commonly used for treatment and followup in patients with asthma. It shows whether the treatment of a patient is under control, and the disease has a satisfactory or unsatisfactory course, measuring the disease status within the last four weeks. It is easily understood by patients and their families and comprises five questions. Patients score each question from 1 to 5 points. The total score of five questions indicates the test result as follows: a score of 25 points is considered as total control, scores from 20 to 25 points as partial control, and a score equal to or below 19 points shows lack of control $[10,11]$. In patients in whom allergic rhinitis and asthma were associated, ACT was performed before initiation of treatment and recorded. ACT was performed again following three months of treatment and recorded. The ACT results before treatment were compared to those after three months of treatment.

\section{Statistical Analysis}

Descriptive statistical methods were used together with the Independent Samples and Paired Samples T-tests for intra-group and intergroup comparisons. The results were evaluated at the confidence interval of $95 \%$, and $p<0.05$ was considered statistically significant. 


\section{RESULTS}

Among 53 patients with allergic rhinitis included in the study, 28 (52.8\%) patients were diagnosed with asthma as an accompanying disorder. The demographic characteristics, smoking and family history, the laboratory test results of the AR patient groups with asthma (AR+AST+) and without asthma (AR+AST-) were shown in Table 1 together with their pulmonary function test results before and after three months of treatment. The mean age of the (AR+AST+) and (AR+AST-) groups were $33 \pm 9$ years and $32 \pm 9$ years, respectively. There was no statistically significant difference between the two groups in terms of gender, age, smoking and family asthma history (p: 0.524, 0.377, 0.609 and 0.359 respectively)
Smoking was absent in most patients in both groups [25/28 (89\%) and 22/25 (88\%), respectively], and the two groups were similar when compared for smoking history $(p=0.609)$.

When the effect of smoking on the anti-histaminic use was investigated, it was found that, in both the $(\mathrm{AR}+\mathrm{AST}+)$ and (AR+AST-) groups, being a smoker or nonsmoker did not have any effect on abandoning or continuing anti-histaminic drugs $(\mathrm{p}=0.377$ and $p=0.477$, respectively). The family history involved the presence of asthma in $61 \%$ and $52 \%$ of the patients in the (AR+AST+) and (AR+AST-) groups, respectively. There was no statistically significant difference between the two groups regarding the presence of asthma in family history $(p=0.359)$.

Table 1. The demographic characteristics, smoking and family history, the laboratory test results, and pulmonary function test results of the patient groups with allergic rhinitis associated with asthma (AR+AST+) and without asthma (AR+AST-).

\begin{tabular}{|c|c|c|c|}
\hline Variable & Asthma (+) & Asthma (-) & $p$ \\
\hline n (\%) & 28 & 25 & - \\
\hline Age (years) mean \pm SD (min-max) & $33 \pm 9(18-52)$ & $32 \pm 9(20-51)$ & 0,524 \\
\hline \multicolumn{4}{|l|}{ Gender } \\
\hline Male/ Female n (\%) & $7 / 21(25 / 75)$ & $10 / 15(40 / 60)$ & 0,377 \\
\hline Smoking (+) n (\%) & $3(11)$ & $3(12)$ & 0,609 \\
\hline Family history (+) for asthma n (\%) & $17(61)$ & $13(52)$ & 0.359 \\
\hline Mite skin prick test (+) n (\%) & $28(100)$ & $25(100)$ & 0.254 \\
\hline Total lgE - mean \pm SD (min-max) & $374 \pm 474(4-2500)$ & $376 \pm 421(3-1507)$ & 0.393 \\
\hline Phadiatop + n (\%) & $18(64)$ & $13(52)$ & 0.314 \\
\hline \multicolumn{4}{|l|}{ Before treatment } \\
\hline \multicolumn{4}{|l|}{ Pulmonary Function tests } \\
\hline FEV1 (mean \pm SD) & $2.96 \pm 0.65$ & $3.35 \pm 1.14$ & 0.020 \\
\hline PEFL (mean \pm SD) & $5.94 \pm 1.79$ & $7.04 \pm 3.04$ & 0.038 \\
\hline PEF (\%) (mean \pm SD) & $78.81 \pm 19.25$ & $84.20 \pm 21.95$ & 0.887 \\
\hline FVC (mean \pm SD) & $88.58 \pm 12.33$ & $90.28 \pm 12.19$ & 0.659 \\
\hline FEV1/FVC (mean \pm SD) & $87.41 \pm 8.68$ & $87.56 \pm 5.49$ & 0.179 \\
\hline ACT score (mean \pm SD) & $16.61 \pm 4.65$ & - & \\
\hline \multicolumn{4}{|l|}{ Following 3-month treatment } \\
\hline \multicolumn{4}{|l|}{ Pulmonary Function tests } \\
\hline FEV1-3Month (mean \pm SD) & $2.94 \pm 0.65$ & $3.28 \pm 0.81$ & 0.054 \\
\hline PEFL-3Month (mean \pm SD) & $6.08 \pm 1.70$ & $10.15 \pm 16.17$ & 0.076 \\
\hline PEF-3Month (\%) (mean \pm SD) & $80.21 \pm 19.10$ & $82.71 \pm 24.46$ & 0.412 \\
\hline FVC-3Month (mean \pm SD) & $86.43 \pm 11.89$ & $89.18 \pm 19.14$ & 0.872 \\
\hline FEV1/FVC3Month(mean \pm SD) & $86.75 \pm 7.81$ & $86.48 \pm 27.07$ & 0.309 \\
\hline Antihistamine use (+) n (\%) & $5(18)$ & $5(20)$ & 0.559 \\
\hline ACT score (mean \pm SD) & $21.46 \pm 3.67$ & - & - \\
\hline
\end{tabular}

*FEV1: Forced expiratory volume in one second, PEFL: Peak expiratory flow liter, FVC: Forced vital capacity, PEF: Peak expiratory flow, ACT: asthma control test 
In all patients of both groups, the mite skin prick test was positive. The phadiatop positivity was determined in $64 \%$ and $52 \%$ of the (AR+AST+) and (AR+AST-) groups, respectively; when the two groups were compared, no statistically significant difference was present between the two groups regarding phadiatop positivity $(p=0.314)$. The mean, the minimum and the maximum values of total Ig E were $374 \pm 474$ (4-2500) and $376 \pm 421$ (3-1507) in the (AR+AST+) and (AR+AST-) groups, respectively. No statistically significant difference was present between the two groups regarding the total Ig E level $(p=0.393)$.

In the group with asthma, the pre-treatment FEV1 and peak expiratory flow liter (PEFL) values of the pulmonary function tests were statistically significantly lower than the group without asthma $(p=0.020$ and $p=0.038$, respectively). There was no statistically significant difference between the two groups in terms of pre-treatment peak expiratory flow (PEF), force vital capacity (FVC) and FEV1 / FVC values. There was no statistically significant difference in pulmonary function test parameters between the two groups after immunotherapy (Table 1).

The two groups were similar regarding their PEF, FVC, and FEV1/FVC values obtained before treatment $(P=0.887, p=0.659$, and $p=0.179$, respectively)
The pulmonary function test results obtained after three months of SCIT in both groups were: FEV1 was $2.94 \pm 0.65$ and $3.28 \pm 0.81$, PEFL was $6.08 \pm 1.70$ and $10.15 \pm 16.17$, PEF was $80.21 \pm 19.10$ and 82.71 \pm 24.46 , FVC was $86.43 \pm 11.89$ and $89.18 \pm 19.14$, and FEV1/FVC was $86.75 \pm 7.81$ and $86.48 \pm 27.07$ in the (AR+AST+) and (AR+AST-) groups, respectively. When the two groups were compared regarding the sub-parameters of pulmonary function tests after three months of SCIT, no significant difference was present between the (AR+AST+) and (AR+AST-) groups regarding their FEV1, PEFL, PEF, FVC, and FEV1/FVC values $(p=0.054, p=0.076, p=0.412$, $p=0.872$, and $p=0.309$, respectively).

In the group without asthma, no statistical difference was present between the pre- and posttreatment FEV1,PEFL, PEF, FVC, and FEV1/FVC values of pulmonary function tests $(p=0.633, p=0.356$, $p=0.756, p=0.805$, and $p=0.401$, respectively) (Table 2, Figure 1). Also, there was no statistically significant difference between pulmonary function tests before and after treatment in the group with asthma. However, the score values of the asthma control test before and after treatment were $16.61 \pm 4.65$ and $1.46 \pm 3.67$. The difference between these two scores was statistically significant $(p=0.000)$ (Table 3) (Figure 2).

Table 2. The comparison of the results of pulmonary function tests performed before and following three months of immunotherapy in the patient group with allergic rhinitis but without asthma (AR+AST-)

\begin{tabular}{|l|c|c|c|}
\hline Variable & $\begin{array}{c}\text { Before initiation of } \\
\text { immunotherapy }\end{array}$ & $\begin{array}{c}\text { Following 3-month } \\
\text { immunotherapy }\end{array}$ & $p$ \\
\hline FEV1 (mean \pm SD) & $3.35 \pm 1.14$ & $\mathbf{3 . 2 8} \pm 0.81$ & 0.633 \\
PEFL (mean \pm SD) & $7.04 \pm 3.04$ & $10.15 \pm 16.17$ & 0.356 \\
PEF (\%) (mean \pm SD) & $\mathbf{8 4 . 2 0 \pm 2 1 . 9 5}$ & $\mathbf{8 2 . 7 1} \pm 24.46$ & 0.756 \\
FVC (mean \pm SD) & $\mathbf{9 0 . 2 8 \pm 1 2 . 1 9}$ & $\mathbf{8 9 . 1 8} \pm 19.14$ & 0.805 \\
FEV1/FVC (mean \pm SD) & $\mathbf{8 7 . 5 6} \pm 5.49$ & $\mathbf{8 6 . 4 8} \pm 27.07$ & 0.401 \\
\hline
\end{tabular}

*FEV1: Forced expiratory volume in one second, PEFL: Peak expiratory flow liter, FVC: Forced vital capacity, PEF: Peak expiratory flow

Table 3. The comparison of the results of pulmonary function tests and the Asthma Control Test performed before and following three months of immunotherapy in the patient group with allergic rhinitis and asthma (AR+AST+)

\begin{tabular}{|l|c|c|c|}
\hline Variable & $\begin{array}{c}\text { Before initiation of } \\
\text { immunotherapy }\end{array}$ & $\begin{array}{c}\text { Following 3-month } \\
\text { immunotherapy }\end{array}$ & $p$ \\
\hline FEV1 (mean \pm SD) & $2.96 \pm 0.65$ & $2.94 \pm 0.65$ & 0.809 \\
PEFL (mean \pm SD) & $5.94 \pm 1.79$ & $6.08 \pm 1.70$ & 0.747 \\
PEF (\%) (mean \pm SD) & $78.81 \pm 19.25$ & $80.21 \pm 19.10$ & 0.833 \\
FVC (mean \pm SD) & $88.58 \pm 12.33$ & $86.43 \pm 11.89$ & 0.456 \\
FEV1/FVC (mean \pm SD) & $\mathbf{8 7 . 4 1 \pm 8 . 6 8}$ & $86.75 \pm 7.81$ & 0.349 \\
Asthma Control Test score (mean \pm SD) & $16.61 \pm 4.65$ & $21.46 \pm 3.67$ & 0.000 \\
\hline
\end{tabular}

*FEV1: Forced expiratory volume in one second, PEFL: Peak expiratory flow liter, FVC: Forced vital capacity, PEF: Peak expiratory flow 
When the pretreatment values of PFT subparameters were compared to those obtained after three months of treatment in the (AR+AST+) group, no statistically significant differences were present for FEV1, PEFL, PEF, FVC, and FEV1/FVC $(p=0.809, p=0.747, p=0.833, p=0.456$, and $p=0.349$, respectively) (Table 3) (Figure 1 ).

Only five patients (18\%) in the (AR+AST+) group, and five (20\%) in the (AR+AST-) group were determined to continue receiving their anti-histaminic drugs, and no difference was present between the two groups regarding the discontinuation rate of antihistaminic treatment $(p=0.559)$. Following allergenspecific immunotherapy, $81.1 \%$ (43) patients involved in the study were found to abandon their anti-histaminic treatments totally.

No patient in both groups was determined to encounter a severe event such as a severe allergic reaction or an episode of asthma during their three months of immunotherapy.

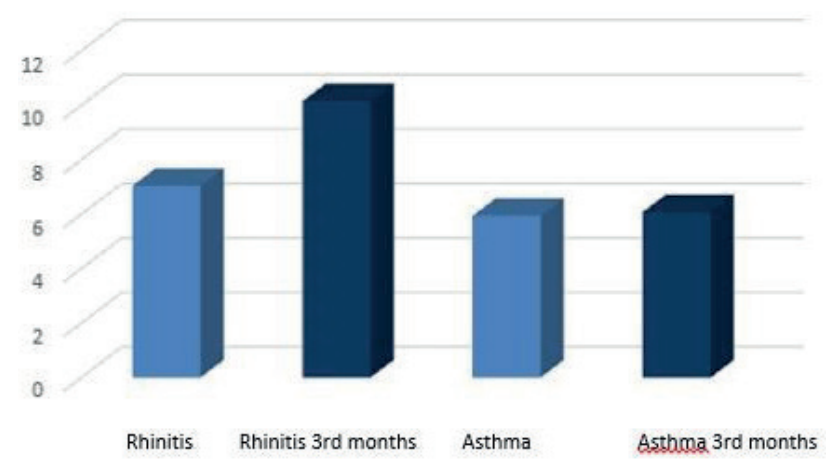

Figure 1. PEFL values at the beginning and 3rd month of treatment in rhinitis patients with and without asthma

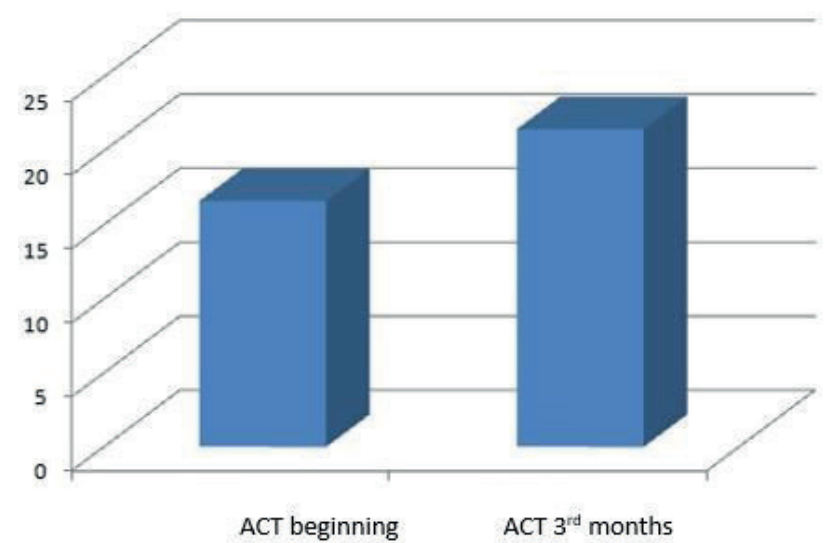

Figure 2. Results of the beginning and 3rd months asthma control test (ACT) in asthmatic patients

\section{DISCUSSION}

Allergic rhinitis and asthma are common chronic disorders affecting over 300.000 .000 people throughout the world. It has been predicted that asthma will affect an additional 100.000 .000 people in 2025 [12]. While allergic rhinitis can mostly be taken under control by anti-histaminic drugs and nasal steroids, most of the patients with asthma can be taken under control by inhaled corticosteroids and montelukast effectively. However, since such treatments cannot handle underlying disorders, relieving symptoms only, symptoms recur with discontinuation of drugs [13, 14]. It has been known that allergen-specific immunotherapy, when administered with repeated and increasing doses, could change the course of allergic diseases by providing immune tolerance $[13,15]$.

Limited data on the relationship between smoking and allergic rhinitis is available. Sözener et al. [16], in their study conducted in Turkey in 2018, determined that $21 \%$ of 252 patients with allergic rhinitis and $11 \%$ of 249 patients with asthma were smokers. In our study, the overall rate of smoking was determined as $11.3 \%(6 / 53)$. The similarity of the smokers and nonsmokers regarding the effect of smoking on treatment response might be related to the small number of smokers in both groups in our study.

In our study, the differences between the pretreatment values of all PFT sub-parameters and the values obtained after three months of SCIT were not significant in both groups $(p<0.05)$ (Tables 2, 3). However, even though it did not have any statistical significance, the PEFL value showed a steep increase from 7.04 to 10.15 with immunotherapy in the patient group having allergic rhinitis only. Such an increase did not occur in the patient group with asthma as a concomitant disease. Ross et al., in their meta-analysis study, evaluated 24 studies on treatment of asthma with and without specific immunotherapy. They concluded that pulmonary functions significantly improved, bronchial challenge was protected against, and the need for medications was reduced when specific immunotherapy was encountered [17]. The results of this meta-analysis study differed from ours; however, the treatment periods of the involved studies were at least one year of duration, much longer than the immunotherapy period of three months in our study. 
The Asthma Control Test (ACT) is currently the most used test, which is easily understood by patients and their families, showing whether asthma has a good or poor prognosis. The aim of treatment in asthma is the provision of total control, and the scoring of ACT shows whether the treatment has been satisfactory $[10,11]$. In a double-blind, placebo-controlled, multicenter study, the efficacies of different doses were investigated in asthma patients receiving mite-specific immunotherapy. The ACT score was determined to be increased in four different treatment groups [18]. In another study that investigated the efficacy of mite-specific SCIT in both asthma and rhinitis using the CARAT (Control of Allergic Rhinitis and Asthma Test) questions at the treatment onset and then at the 4th month of treatment statistically significantly definite clinical improvement was determined [19]. Similar to these studies, in our study also, a statistically strongly significant increase in the ACT score was determined, suggesting that significant clinical improvement of asthma occurred in our patients with asthma following three months of allergen-specific immunotherapy.

The efficacy of immunotherapy is evaluated based on the symptoms, visual analog scale, and drug utilization. In a study investigating the efficacy of immunotherapy in asthma, it was determined that in the 6th month, the dose of inhaled steroids had significantly decreased in the group receiving immunotherapy compared to the non-receiving group [20]. In a Cochrane review of 88 studies, it was determined that, with allergen-specific immunotherapy, the symptoms of asthma and drug utilization were significantly reduced as well as the bronchial hyperactivity [21]. Similar to the results of the studies mentioned above, in our study, $81.1 \%$ (43/53) of allergic rhinitis patients with/without asthma were found to quit receiving anti-histaminic drugs following allergen-specific immunotherapy. Five of ten patients who continued their antihistaminic drugs were asthma patients. Also, in our study, with ACT scoring, clinical improvement of asthma was determined to occur following three months of immunotherapy in our allergic rhinitis patients with asthma.

The limitations of the study were its retrospective nature and its small sample size.

\section{CONCLUSION}

By using pulmonary function tests and Asthma Control Test in allergic rhinitis and asthma, we showed that the effectiveness of allergen specific immunotherapy started in three months, which was earlier when compared to the current literature.

When asthma is associated with allergic rhinitis, which is quite common, the control of asthma is also provided with the treatment of allergic rhinitis. The immunotherapy used to treat allergic rhinitis reduces the requirement for anti-histaminic drugs and takes the accompanying asthma disease under control.

\section{CONFLICT of INTEREST}

The authors declare that they have no conflict of interest. 
[1] Bousquet J, Khaltaev N, Cruz A.A, et al. Allergic Rhinitis and its Impact on Asthma (ARIA) 2008 update (in collaboration with the World Health Organization, GA(2)LEN and AllerGen). Allergy, 2008; 63 Suppl 86: 8-160.

[2] Sakar A, Yorgancioglu A, Dinc G, et al. The prevalence of asthma and allergic symptoms in Manisa, Turkey (A western city from a country bridging Asia and Europe). Asian Pac J Allergy Immunol. 2006; 24(1): 17-25.

[3] Dinmezel S, Ogus C, Erengin H. The prevalence of asthma, allergic rhinitis, and atopy in Antalya, Turkey. Allergy Asthma Proc. 2005; 26(5): 403-9.

[4] Bousquet J, Annesi-Maesano I, Carat F, et al.Characteristics of intermittent and persistent allergic rhinitis: DREAMS study group. Clin Exp Allergy. 2005; 35(6): 728-32.

[5] Antonicelli L, Micucci C, Voltolini S, et al.Allergic rhinitis and asthma comorbidity: ARIA classification of rhinitis does not correlate with the prevalence of asthma. Clin Exp Allergy. 2007; 37(6): 954-60.

[6] Bousquet, J, Vignola AM, Demoly P. Links between rhinitis and asthma. Allergy. 2003; 58(8): 691-706.

[7] Akdis CA, Akdis M. Mechanisms of allergen-specific immunotherapy. J Allergy Clin Immunol. 2011; 127(1):1827.

[8] Asamoah F, Kakourou A, Dhami S, et al. Allergen immunotherapy for allergic asthma: a systematic overview of systematic reviews. Clin Transl Allergy. 2017; 7: 25.

[9] Global Initiative for Asthma. Global Strategy for Asthma Management Prevention. 2019; Avaible from: www. ginaasthma.org

[10] Schatz M, Sorkness CA, Li JT, et al. Asthma Control Test: reliability, validity, and responsiveness in patients not previously followed by asthma specialists. J Allergy Clin Immunol. 2006; 117(3): 549-56.

[11] Nathan RA, Sorkness CA, Kosinski M, et al. Development of the asthma control test: a survey for assessing asthma control. J Allergy Clin Immunol. 2004; 113(1): 59-65.
[12] Bateman ED, Hurd SS, Barnes PJ, et al. Global strategy for asthma management and prevention: GINA executive summary. Eur Respir J. 2008; 31(1): 143-78.

[13] Yukselen A, Kendirli SG. Role of immunotherapy in the treatment of allergic asthma. World J Clin Cases. 2014; 2(12): 859-65.

[14] Tamaoki J, Isono K, Taira M, et al. Role of regular treatment with inhaled corticosteroid or leukotriene receptor antagonist in mild intermittent asthma. Allergy Asthma Proc. 2008; 29(2): 189-96.

[15] James LK, Durham SR. Update on mechanisms of allergen injection immunotherapy. Clin Exp Allergy. 2008; 38(7):1074-88.

[16] Sozener ZC, Ciftci F, Soyyigit S. et al. Smoking attitudes of the patients with allergic rhinitis: a comparison with asthma and chronic obstructive pulmonary disease. Are there differences when only upper airways are involved? Tuberk Toraks. 2018;66(1):43-51.

[17] Ross RN, Nelson HS, Finegold I. Effectiveness of specific immunotherapy in the treatment of asthma: a metaanalysis of prospective, randomized, double-blind, placebo-controlled studies. Clin Ther. 2000; 22(3):329-41.

[18] Jutel M, Rudert $M$, Kreimendahl F, et al. Efficacy and tolerability of a house dust mite allergoid in allergic bronchial asthma: a randomized dose-ranging trial. Immunotherapy. 2018; 10(13): 1149-1161.

[19] Valle-Rodriguez F, Lopez-Garcia Al, Rivero-Yeverino D, et al. [The effectiveness and safety of subcutaneous immunotherapy for inhalable allergens in patients with respiratory allergies]. Rev Alerg Mex. 2019; 66(3): 301-307.

[20] Rhyou HI, Nam YH. Efficacy of Allergen Immunotherapy for Allergic Asthma in Real World Practice. Allergy Asthma Immunol Res. 2020; 12(1):99-109.

[21] Abramson MJ, Puy RM, Weiner JM. Injection allergen immunotherapy for asthma. Cochrane Database Syst Rev. 2010; 8: 01186. 